\title{
Redes de bits como alternativa às redes neurais em problemas de aprendizado por reforço
}

\author{
Nickolas R. Machado ${ }^{1}$, Pedro C. F. Machado ${ }^{1}$, Juliana M N S Zamith ${ }^{1}$, Filipe \\ Braida $^{1}$, Leandro Alvim ${ }^{1}$, Raul S. Ferreira ${ }^{2}$ \\ ${ }^{1}$ Departamento de Ciência da Computação - Universidade Federal Rural do Rio de \\ Janeiro (UFRRJ) - Rio de Janeiro - RJ - Brasil \\ ${ }^{2}$ Departamento de Computação e Robótica - LAAS-CNRS, Toulouse, França \\ Resumo. Redes neurais artificiais (RNAs) são amplamente utilizadas, como \\ por exemplo, em jogos digitais através de agentes inteligentes que replicam o \\ comportamento humano. Contudo, o custo computacional de treinamento \\ delas costuma ser alto, exigindo a escolha entre maior processamento e menor \\ qualidade. Desta forma, este trabalho propõe a aplicação de redes de bits \\ (RBs) como uma alternativa capaz de maximizar o processamento e minimizar \\ o uso de memória. Comparados às RNAs de precisão simples, os resultados \\ mostram um speedup de até 91 vezes usando 32 vezes menos memória.
}

\section{Introdução e Motivação}

Redes neurais são aplicadas com sucesso em problemas da computação. Contudo, o bom desempenho delas exige alto custo computacional, limitando a utilização pelo hardware, principalmente em arquiteturas embutidas. Entre as soluções para este problema estão as redes de bits $(R B)$, uma alternativa que consome menos memória e faz uso de menos instruções que redes de precisão simples (RF32) [1][2].

Isso se deve às $R B s$ substituírem os parâmetros das redes neurais (pesos), que geralmente são números reais em palavras de 32 bits (Float) por bits, o que reduz o espaço necessário para representar a informação, uma vez que um Float pode constituir 32 pesos e não apenas um. Assim, o uso de $R B s$ pode diminuir em até 32x a quantidade de memória utilizada. Ainda, a representação de bits permite trocar vários procedimentos de multiplicação por uma operação bit a bit do tipo XOR [1].

Outro fator relevante é o impacto no treinamento das $R B s$. Tendo em vista que o espaço de busca de uma $R F 32$ é de aproximadamente $\left(2^{32}\right)^{p}$ combinações, onde $p$ é o número de pesos, em redes com quantificação binária, isto é, as que assumem os pesos apenas como valores 0 ou 1 representando respectivamente +1 e -1 , esse monte se reduz a $2^{p}$ [1]. Logo, o tempo gasto para encontrar uma das soluções é menor.

Contudo, a retirada dos números reais leva à perda de precisão da rede, podendo ser necessário ter mais neurônios e/ou aplicá-la apenas a algumas classes de problemas onde não é necessária alta exatidão na entrada. Um exemplo disto é o jogo Snake.

Este trabalho tem como objetivo estudar e verificar o impacto da quantificação binária, na entrada e nos pesos, e o uso de bits no tempo de execução e no consumo de memória durante o andamento da aplicação. Além disso, dois cenários de testes foram implementados para uma análise comparativa entre $R B s$ e $R F 32 s$ : um deles retrata o 
desempenho das redes com entradas e pesos aleatórios, e outro com elas aplicadas ao treinamento no Snake. A próxima seção apresenta detalhes da implementação destes casos, bem como um algoritmo genético [3] utilizado no treinamento.

\section{Implementação do jogo Snake utilizando redes de bits}

$\mathrm{Na}$ implementação do Snake deste trabalho, o usuário, neste caso uma rede neural, deve mover a cobra pelo cenário em busca da comida. Ainda, quando a cabeça da cobra (head) encontra um alimento, o tamanho dela é acrescido e a pontuação aumentada, e ao encostar em uma parte do corpo ou em uma parede, há derrota. Além disso, a posição inicial dos alimentos foi fixada para criar um contexto controlado de treinamento. Outrossim, a cobra trata-se de um agente que possui 20 sensores fornecendo dados binários, do tipo está e não está, calculados com relação às posições da head e das 4 direções (norte, sul, oeste, leste), localizando a comida, partes do corpo e as paredes.

A $R B$ e $R F 32$ utilizadas possuem 20 neurônios na camada oculta, escolhidos para que a rede possa elaborar de estratégias complexas no jogo, e 4 na camada de saída para os possíveis movimentos da cobra. Ademais, a implementação foi feita em Julia por ser uma linguagem com características de alto nível e foco em desempenho. Por fim, foram usados vetores do tipo UInt64 nos pesos das RBs que possibilitam o armazenamento e execução de 64 bits através de uma única operação.

Para o treino das redes foi feita uma versão do algoritmo genético com seleção elitista, crossover com corte em ponto, mutação de bias e pesos e avaliação [3] baseada nas comidas coletadas, distância do alimento e movimentos que o afastam deste último.

\section{Testes}

Os testes descritos nesta seção foram realizados em um Core $i 78750$ h Intel de 6 núcleos e 12 threads e $16 G B$ de memória $D D R 4$. O primeiro teste consistiu em avaliar a performance da $R B$. Para tanto, foi usado um vetor de valores aleatórios como entrada de dados e 512 neurônios com pesos também aleatórios. Ainda, foram implementadas três versões do código: a primeira delas, chamada $\mathrm{V}_{\mathrm{Bit}}$, que utiliza $R B s$; a segunda variante, $\mathrm{V}_{\mathrm{FI} \text {.3), }}$, constitui-se de forma similar à $\mathrm{V}_{\mathrm{Bit}}$, mas operando com $R F 32 \mathrm{~s}$; e por fim a $\mathrm{V}_{\text {Flux }}$, que foi implementada empregando o Flux ${ }^{1}$, uma biblioteca para machine learning desenvolvida em Julia.

A Tabela 1 apresenta o tempo de execução e a quantidade de memória consumida nas três versões. Variou-se o tamanho da entrada para avaliar o impacto desta no desempenho. Com isso, é visto através da tabela que o consumo médio aproximado de memória da rede de bits é 32x menor quando comparada com os outros dois casos. Quanto ao tempo de execução, verificando as variantes $\mathrm{V}_{B i t}$ e $\mathrm{V}_{F L 32}$ foi obtido um speedup de até $91 \mathrm{x}$ nos testes. Contudo, quando comparadas as versões $\mathrm{V}_{B i t}$ e $\mathrm{V}_{\text {Flux }} \mathrm{o}$ maior speedup médio foi em torno de 10x. Acredita-se que esta diferença decorre das otimizações proporcionadas pelo $B L A S^{2}$, uma biblioteca para álgebra linear com diversos aprimoramentos matemáticos e de uso dos recursos de hardware, empregada pelo Flux na execução das RF32s.

\footnotetext{
${ }^{1}$ Flux, https://fluxml.ai/.

${ }^{2}$ BLAS, http://www.netlib.org/blas/
} 
Tabela 1. Quantidade de memória utilizada, tempo de execução e speedup obtidos para uma média de 600 execuções.

\begin{tabular}{|c|c|c|c|c|c|c|c|c|}
\hline \multirow{2}{*}{ Entrada } & \multicolumn{3}{|c|}{ Tempo $(\mu \mathrm{s})$} & \multicolumn{3}{c|}{ Tamanho (MB) } & \multicolumn{3}{c|}{ Speedup } \\
\cline { 2 - 9 } & $\mathrm{V}_{\text {Bit }}$ & $\mathrm{V}_{F L 32}$ & $\mathrm{~V}_{F l u x}$ & $\mathrm{~V}_{\text {Bit }}$ & $\mathrm{V}_{F L 32}$ & $\mathrm{~V}_{F l u x}$ & $\mathrm{~V}_{F L 32 \times \text { Bit }}$ & $\mathrm{V}_{\text {Flux } x \text { Bit }}$ \\
\hline 512 & 5.542 & 283.343 & 14.761 & 0.031 & 1.000 & 1.002 & 51.126 & 2.663 \\
\hline 4096 & 31.646 & 2402.852 & 123.680 & 0.250 & 8.000 & 8.002 & 75.929 & 3.908 \\
\hline 16384 & 105.387 & 9667.600 & 1059.619 & $\mathbf{1 . 0 0 0}$ & $\mathbf{3 2 . 0 0 0}$ & $\mathbf{3 2 . 0 0 2}$ & $\mathbf{9 1 . 7 3 4}$ & $\mathbf{1 0 . 0 5 4}$ \\
\hline
\end{tabular}

$\mathrm{O}$ segundo teste consistiu em avaliar o desempenho das $R B s$ no treinamento aplicado ao Snake. Os resultados obtidos são apresentados no Gráfico 1. Neste, é possível perceber uma considerável redução no número de gerações obtidas na versão que usa $R B s\left(\mathrm{~V}_{\mathrm{Bit}}\right)$ comparado com a outra que utiliza $R F 32 s\left(\mathrm{~V}_{\text {Flux }}\right)$. Contudo, o tempo não diminuiu como esperado. Isto se deve à computação da avaliação não ser o mais custoso no treinamento, e a mutação de bits requerer mais operações para ser realizada.

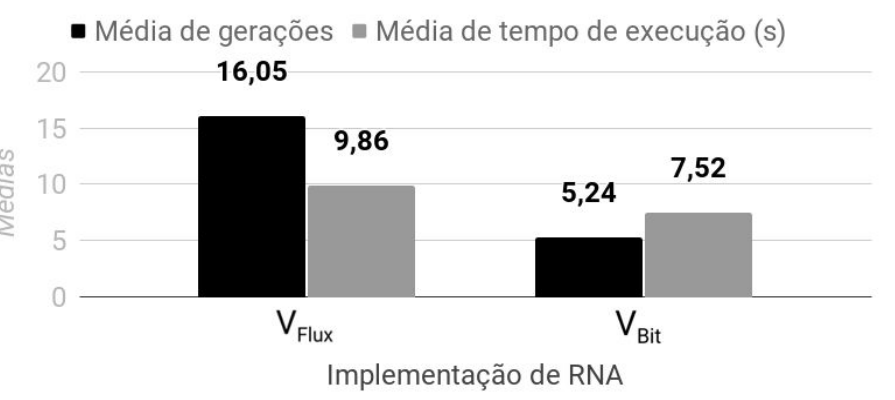

Gráfico 1. Comparação média de 100 execuções entre redes de bits e Float32 na convergência do jogo Snake até atingir o placar mínimo de 63 pontos na avaliação.

\section{Conclusão}

Através dos testes é possível perceber que a execução em $R B s$ é mais rápida que em $R F 32 s$, enquanto nem sempre essa melhora é expressiva no treinamento. Contudo, dependendo do uso dessas redes na avaliação e o impacto dos pesos binários no treinamento, o speedup neste pode ser um fator decisivo para usá-las. Ainda, mesmo que haja um treinamento demorado, o consumo de memória e o desempenho das $R B S$ oportuniza o emprego delas em $C P U$, viabilizando uma gama de usos para elas.

\section{Referências}

[1] Rastegari, M., Ordonez, V. , Redmon, J. and Farhadi, A. (2016) "XNOR-Net: ImageNet Classification Using Binary Convolutional Neural Networks" https://arxiv.org/pdf/1603.05279.pdf, Agosto.

[2] Assis, P. (2009) “O que são Redes Neurais?” https://bit.ly/3mqTBN2, Setembro.

[3] Massago, S. (2013) “Introdução ao Algoritmo Genético" https://cutt.ly/GhfdpJL, Agosto. 